\title{
Optical triggered seizures using a caged 4-Aminopyridine
}

\section{Mingrui Zhao ${ }^{1 *}$, Laura M. McGarry ${ }^{2}$, Hongtao Ma ${ }^{1}$, Samuel Harris ${ }^{1,3}$, Jason Berwick ${ }^{3}$, Rafael Yuste $^{2 *}$ and Theodore H. Schwartz ${ }^{1 *}$}

${ }^{1}$ Department of Neurological Surgery, Brain and Mind Center, New York Presbyterian Hospital, Weill Medical College of Cornell University, New York, NY, USA

${ }^{2}$ Department of Biological Sciences, Columbia University, New York, NY, USA

${ }^{3}$ Department of Psychology, University of Sheffield, Sheffield, UK

\section{Edited by:}

Jorge J. Riera, Florida International

University, USA

Reviewed by:

Anna W. Roe, Vanderbilt University, USA

Jean-Christophe Sandoz, Centre

National de la Recherche

Scientifique, France

\section{*Correspondence:}

Mingrui Zhao and Theodore $\mathrm{H}$.

Schwartz, Department of

Neurological Surgery, Weill Medical

College of Cornell University, 525

East 68th Street, Box 99, New York,

NY 10065, USA

e-mail:miz2003@med.cornell.edu;

schwarh@med.cornell.edu;

Rafael Yuste, Department of

Biological Sciences and

Neuroscience, Columbia University,

New York, NY 10027, USA

e-mail: rmy5@columbia.edu
Animal models of epilepsy are critical not only for understanding the fundamental mechanism of epilepsy but also for testing the efficacy of new antiepileptic drugs and novel therapeutic interventions. Photorelease of caged molecules is widely used in biological research to control pharmacologic events with high spatio-temporal resolution. We developed a technique for in vivo optical triggering of neocortical seizures using a novel caged compound based on ruthenium photochemistry (RuBi-4AP). Epileptiform events in mouse cortex were induced with blue light in both whole brain and focal illumination. Multi-electrode array recording and optical techniques were used to characterize the propagation of these epileptic events, including interictal spikes, polyspikes, and ictal discharges. These results demonstrate a novel optically-triggered seizure model, with high spatio-temporal control, that could have widespread application in the investigation of ictal onset, propagation and to develop novel light-based therapeutic interventions.

Keywords: caged compound, epilepsy model, neocortex, electrophysiology, optical imaging, photostimulation

\section{INTRODUCTION}

Epilepsy is a disease that affects about $1 \%$ of the population in the United States involving recurrent seizures, or ictal events (Thurman et al., 2011). The lifelong effects of chronic epilepsy can be devastating due to progressive cognitive decline from short duration electrical discharges that occur throughout the epileptic network between seizures called interictal events. Moreover, uncontrolled seizures cause increases in patient morbidity and mortality. The cost to society is enormous (Begley et al., 2000; Wilner et al., 2014). The mechanism of neocortical epilepsy is poorly understood (Navarro et al., 2002). Animal models of epilepsy are very important not only for understanding the fundamental mechanisms of epilepsy but also for testing the efficacy of new antiepileptic drugs or other therapeutic interventions (Löscher, 2011). New neuroimaging and neuro-modulatory techniques, such as optogenetics have emerged as a popular tool to probe and control neuronal activity with light. Recently, focal seizure-like afterdischarges have been induced in rat hippocampus using optogenetics (Osawa et al., 2013). Caged compounds, also called phototriggers, are widely used in biological research. Photorelease of caged molecules can be used to administer neurotransmitters and other chemicals with high spatial-temporal resolution. However, the ability of such caged compounds to trigger seizures and interictal events has not been previously investigated.

4-Aminopyridine (4-AP) is a relatively selective blocker of voltage-activated $\mathrm{K}^{+}$channels and a potent convulsant when applied to the neocortex. Focal 4-AP application to the neocortex in vivo generates tonic-clonic ictal focal electrographic seizures, which respond to all standard anticonvulsants (Bruckner and Heinemann, 2000). In the in vitro slice model, 4-AP is released into the bath solution, thus influencing the whole slice in a generalized way. With bath application, often in association with removal of magnesium from the bath (Yang et al., 2010), seizurelike events can initiate in multiple locations and multiple layers, but in an uncontrolled fashion. In vivo, on the other hand, microinjection of 4-AP can induce focal seizures, but the volume of tissue in which the 4-AP is released is difficult to control and seizures initiate from only one location. The ability to noninvasively induce focal seizures in restricted areas of cortex with precise control of the timing of these events does not currently exist either in vivo or in vitro.

Caged compounds consist of a chemical entity composed of two parts: the caged compound of interest and a cage moiety that inhibits its action (Mayer and Heckel, 2006). Upon irradiation, the compound of interest is freed and can interact with the surrounding media. A spatial resolution of $<0.5 \mu \mathrm{m}$ and a temporal resolution of ns-to-ms are ideally achievable with focal fast photolysis. Recently, a new group of caged compound based on metal coordination chemistry was developed to study brain function (Rial Verde et al., 2008; Salierno et al., 2010; Araya et al., 2013). Ruthenium bipyridyl complexes can undergo ligand substitution when irradiated with visible light, without the production of a radical species. 
In this manuscript we examine and describe a new technique for triggering seizures and interictal spikes used caged 4-AP (Nikolenko et al., 2005). This new technique, in contrast to the technique previously employed in our laboratory, namely focal injection of 4-AP (Zhao et al., 2011), allows the possibility of precise spatiotemporal control of the initiation and intensity of the ictal or interictal event allowing the investigator to address a variety of new questions with increased experimental control.

\section{MATERIALS AND METHODS ANIMAL PREPARATION}

All experimental procedures were approved by the Weill Cornell Medical College Animal Care and Use Committee following NIH guidelines. Adult male CD1 mice (6-8 weeks) were anesthetized and maintained stable at normal values as previously described (Zhao et al., 2009).

\section{ACUTE EPILEPSY MODEL}

Adult CD1 mice were used for these experiments after approval was obtained by the IACUC of Weill Cornell Medical College. Mice were induced with isoflurane (2-4\%) in $70 \% \mathrm{~N}_{2}: 30 \% \mathrm{O}_{2}$ by facemask. After induction, the animal was maintained under isoflurane anesthesia using a small mask at $\sim 1.5 \%$. Animals were mounted in a stereotaxic frame. The heart rate, arterial blood oxygen saturation, and end-tidal $\mathrm{CO}_{2}$ were monitored and maintained at normal values throughout the experiment. Temperature was monitored rectally and maintained at $37^{\circ} \mathrm{C}$ with a homeothermic blanket system (Harvard Apparatus, Holliston MA). A $\sim 4 \times 5 \mathrm{~mm}$ cranial window was opened over one hemisphere between Lambda and Bregma to expose the neocortex. The dura was carefully removed. Ruthenium-bipyridinetriphenylphosphine caged 4-aminopyridine (RuBi-4-AP) $(200 \mu \mathrm{l}$, $10 \mathrm{mM}$ ) was topically applied or microinjected into neocortex at least $30 \mathrm{~min}$ before uncaging. For the focal illumination, $2 \mu \mathrm{l}$ RuBi-4-AP solution was injected in neocortex at the depth of $300 \mu \mathrm{m}$ using an UltraMicroPump (UMP-3, WPI, Sarasota, FL) via a glass electrode controlled at a speed of $100 \mathrm{nl} / \mathrm{min}$. For control experiments, 4-AP ictal discharges were induced by injecting 4-AP (Sigma, $15 \mathrm{mM}, 0.5 \mu \mathrm{l}$ ) at the same location using a Nanoject II injector as previously described (Schwartz and Bonhoeffer, 2001; Zhao et al., 2009).

\section{PHOTOSTIMULATION}

Rub-4-AP can be uncaged with either 1 photon excitation $(\lambda=$ $480 \mathrm{~nm}$ ) or 2-photon excitation (800 nm) (Nikolenko et al., 2005; Fino et al., 2009). Uncaging was performed with $470 \mathrm{~nm}$ LED light (Figure 1A, Thorlabs, Newton, NJ) either broadly to the entire cortex or focally, delivered through an optical fiber (OD: $200 \mu \mathrm{m}$, Thorlabs, Newton, NJ), using a high power LED driver (LEDD1B, Thorlabs, Newton, NJ), controlled by a stimulator (Master-8; AMPI, Jerusalem, Israel). Optical power delivered at the fiber tip was calibrated with a PM100USB power meter (Thorlabs, Newton, NJ).

\section{IN VIVO ELECTROPHYSIOLOGY}

A single channel local field potential (LFP) was recorded by placing a glass electrode (impedance, $2-4 \mathrm{M} \Omega$ ) filled with $0.9 \%$ saline into the cortex at an acute $\sim 45^{\circ}$ angle. The LFP was amplified and filtered between 0.1 and $1000 \mathrm{~Hz}$ using an AC/DC Differential Amplifier (Model 3000, A-M Systems, Carlsborg, WA). The signal was then digitized by a CED Power 1401 (Cambridge Electronic Design, Cambridge UK), and recorded onto a PC using Spike as previously described (Zhao et al., 2009). In some experiments, multi-channel depth electrodes were employed for multi-layer recordings (16 channels with $100 \mu \mathrm{m}$ spacing, site area $177 \mu \mathrm{m}^{2}, 1.5-2.7 \mathrm{M} \Omega \mathrm{s}$ impedance, and $33 \mu \mathrm{m}$ tip width; Neuronexus Technologies, Ann Arbor, MI), coupled to a preamplifier and data acquisition device (RZ5D workstation, TDT, Alachua, FL) using RPvdsEx software (TDT, Alachua, FL) (Harris et al., 2013).

\section{INTRINSIC OPTICAL SPECTROSCOPIC IMAGING}

The exposed cortical surface was illuminated with one of two different wavelengths using two high power LEDs either at $530 \mathrm{~nm}$ (M530L2; $1600 \mathrm{~mA}$;Thorlabs, Newark, NJ) or $780 \mathrm{~nm}$ (M780L2, 1000 mA; Thorlabs, Newark, NJ). The light was provided through a liquid light-guide placed $\sim 20 \mathrm{~mm}$ away from the cortex. Reflected light was collected through a tandem lens assembly using two $50 \mathrm{~mm}$ camera lenses. Signals recorded at $530 \mathrm{~nm}$, an isosbestic wavelength for hemoglobin, provide a measure of total hemoglobin ( $\mathrm{Hbt}$ ), which is proportional to cerebral blood volume (CBV) if the hematocrit remains stable (Grinvald et al., 1986; Frostig et al., 1990). At $780 \mathrm{~nm}$, the majority of the optical signal is derived from the scattering of light caused by a variety of factors including cell swelling and intra- and extra-cellular fluid shifts, which provide an indirect spatial representation of neuronal activity (Salzberg et al., 1985). Images were acquired at $10 \mathrm{~Hz}$ (Imager 3001, Optical Imaging Inc.) as previously described (Zhao et al., 2011). A TTL signal from each image is routed to an Arduino microcontroller (Arduino Diecimila, Sparkfun Electronics, Boulder, CO), which is programmed to serially strobe the two LED drivers, alternating between the two wavelengths.

\section{DATA ANALYSIS}

Offline analysis was performed using custom analysis software written in Matlab (MathWorks, Natick, MA). Reflectance changes at 530 and $780 \mathrm{~nm}$ were expressed as a negative percentage change from the baseline $(-\Delta \mathrm{R} / \mathrm{R})$. Each frame following the onset of each seizure, measured with the LFP, and for $20 \mathrm{~s}$ before onset, was divided by an average of the 20 frames $(2 \mathrm{~s})$ prior to the onset. An average of the percent fractional change in the optical signal, positive peak, and negative peak at each time point were used for further analysis. The time interval between the ictal onset and the time point where the optical signal crossed 2SD beyond the baseline recording was used to measure the latency of optical signal. For epileptic events, total LFP ( $\Sigma$ LFP) power was calculated as an integral of the area above 2 SD baseline activity under the response curve of each event. Baseline activity was measured over a $2 \mathrm{~s}$ epoch prior to the initiating spike for epileptic events or $10 \mathrm{~s}$ before/after light illumination. A longer window was used prior to illumination since the changes would be more subtle and this would permit an increase in the signal to noise ratio. 

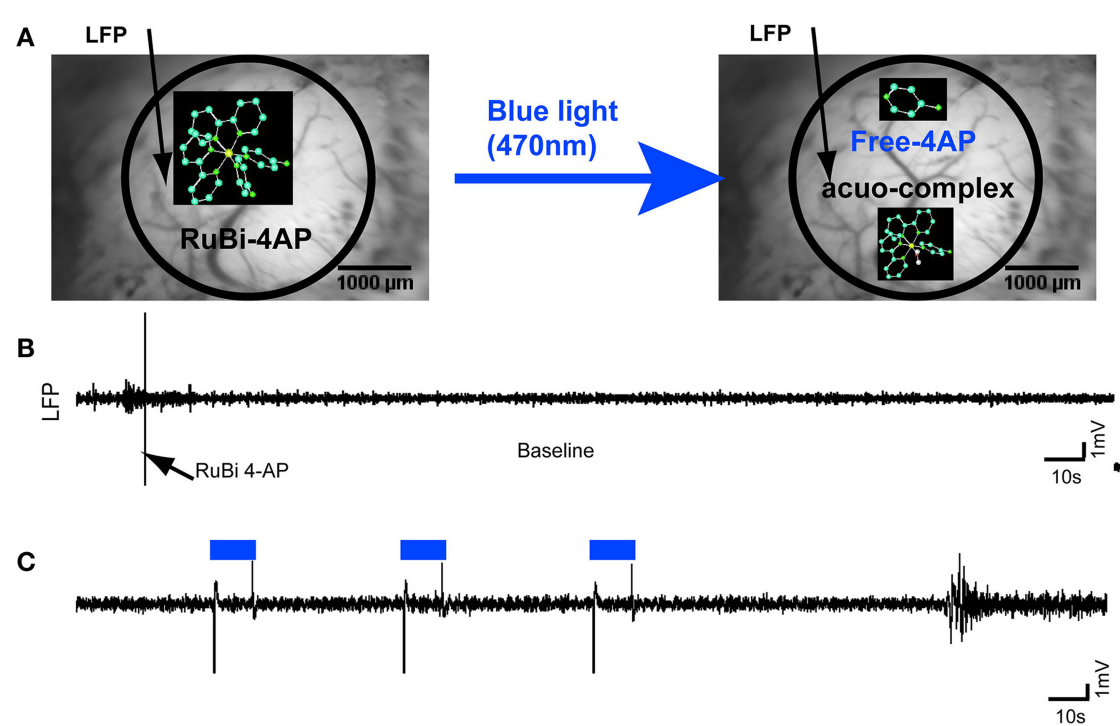

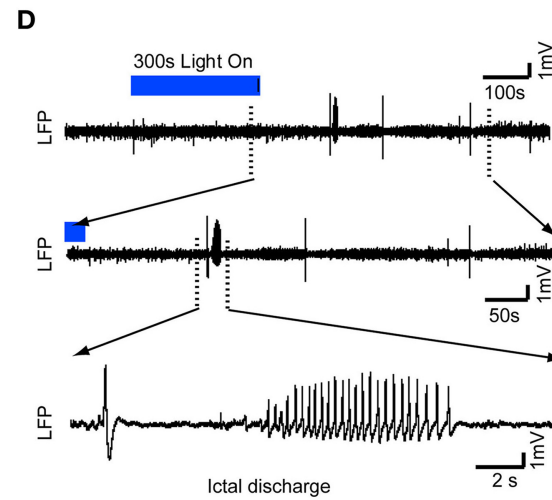

FIGURE 1 | Optical triggering of epilepsy using topical application of RuBi-4-AP in vivo. (A) Schematic diagram of experimental design. RuBi-4-AP $(0.1 \mathrm{ml}, 15 \mathrm{mM})$ is topically applied to induce seizures. RuBi-4-AP is stable in ACSF solution (Left) and the 4-AP and ruthenium complex are photoreleased with visible $470 \mathrm{~nm}$ blue light (Right). The black arrow on the image of the cortical surface indicates the LFP electrode. (B) $5 \mathrm{~min}$ baseline LFP recording shows normal brain activity after RuBi-4-AP application before uncaging. (C) Interictal discharges and
E

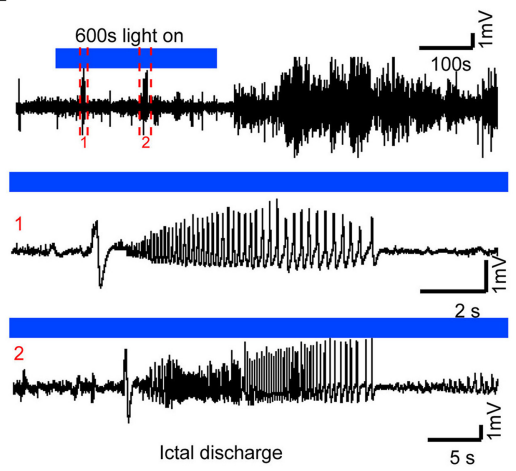

polyspikes are induced by $10 \mathrm{~s}$ blue light illumination $(470 \mathrm{~nm}, 1.2 \mathrm{~A})$ over the neocortex. The duration of illumination is shown with a blue line (Top). (D) Ictal discharge is induced by $300 \mathrm{~s}$ blue light pulses. The expanded view (Bottom) shows a typical ictal discharge starting with an initial spike (ictal onset). (E) Ictal-like activity is also induced by longer, $600 \mathrm{~s}$, illumination. Ictal discharges are recorded during illumination and even after the blue light is turned off (Top). The middle (1) and bottom panels (2) show expanded views of two ictal discharges in the upper panel.
For all data, statistical significance was determined with Student's $T$-test. All data were expressed as means \pm standard error of mean (SEM).

\section{RESULTS}

\section{OPTICAL TRIGGERING OF EPILEPSY USING TOPICAL APPLICATION OF RuBi- 4-AP}

4-AP is a potent convulsant (Rogawski and Barker, 1983; Stansfeld et al., 1986; Mattia et al., 1993; Barkai et al., 1995; Benardo, 1997). In previous experiments, we have shown that 4-AP is a powerful convulsant when injected into the neocortex of in vivo anesthetized rat and mouse (De la Cruz et al., 2011; Zhao et al., 2011). In order to test whether the caged compound $\mathrm{RuBi}-4-\mathrm{AP}$ would induce abnormal brain activity prior to photorelease and to ensure that the illumination alone would not elicit abnormal cortical activity, we performed the following control experiments. Once the surgery was complete, we recorded baseline LFP signals for $15 \mathrm{~min}$ to ensure the health of the neocortex and the absence of any abnormal activity that might result from the trauma of surgery. Animals (5\%) with abnormal spikes or bursts induced by surgical damage before RuBi-4-AP application were excluded from the further experiments. Blue light was applied to the cortex, $20 \mathrm{~mm}$ from its surface, for a period of 600 s. No abnormal spikes, bursts or DC shifts were identified. In fact, the illumination appeared to have no effect on the LFP after comparing the power spectrum of the LFP before and after illumination (averaged $\Sigma \mathrm{LFP}_{\text {power }}$ in $10 \mathrm{~s}: 38.57 \pm 21.27 \mathrm{mV}^{2}$ vs. $36.25 \pm 13.83 \mathrm{mV}^{2}, n=4$ mice, $\left.p>0.05\right)$. This control experiment demonstrates that the induced epileptic activity was not caused by light damage or any other photostimulation effect. RuBi-4-AP $(15 \mathrm{mM}, 200 \mu \mathrm{l})$ was then topically applied on the neocortex for $30 \mathrm{~min}$. In the absence of blue light illumination 
(no uncaging), there were no abnormal spikes, bursts or DC shifts detected and no significant change in the power spectrum of the LFP for all recorded mice (Figure 1B). This result indicated that the caged compound was stable without blue light illumination and when not uncaged, did not trigger any type of epileptiform events.

\section{WIDE-FIELD ILLUMINATION}

We used a high power $470 \mathrm{~nm}$ LED light (1.2 A) to uncage RuBi4-AP over an exposed cortical region in anesthetized mice. The duration of illumination could be used to trigger different types of events, either interictal spikes or ictal events. Using short 10s pulses, interictal spikes were reliably induced (Figure 1C). The average time between short-duration illumination and interictal events was $0.90 \pm 0.21 \mathrm{~s} \quad(n=4$ mice, 268 interictal spikes after 120 photostimulations). Following longer duration illumination (300-600s), seizure-like ictal discharges were triggered (Figures 1D,E). These seizures typically began with a large spikeand-wave followed by a low amplitude fast activity, or recruiting rhythm, which evolved into rapid spike-and-wave activity that gradually increased in periodicity and decreased in amplitude until the seizure terminated. Consistent with our previous 4-AP experiments using focal injection of the drug (Ma et al., 2013), some seizures began with low-voltage fast activity without the initial spike. Even after illumination was discontinued, ictal-like events were continuously recorded for up to $3100 \mathrm{~s}$, indicating that the uncaged drug was persistently active and did not become re-caged. The morphology of these events is similar to those elicited with focal 4-AP injection in rat neocortex and also human neocortical seizures (Zhao et al., 2007, 2009). In total, 268 interictal spikes ( $n=4$ mice) and 34 ictal discharges were recorded ( $n=$ 4 mice). The average duration of seizures was $39.41 \pm 11.57 \mathrm{~s}$. The average delay between the onset of long-duration illumination and a first ictal event was $303.10 \pm 151.55 \mathrm{~s}$.

\section{FOCAL ILLUMINATION}

After demonstrating the ability to trigger seizures with a large, diffuse uncaging, we wished to determine if focal uncaging was possible, which would permit spatial control of the site of onset of the seizures. Two $\mu \mathrm{l}$ of $25 \mathrm{mM}$ RuBi-4-AP solution were injected into the neocortex at the depth of $300 \mu \mathrm{m}$ using a MicroPump via a glass electrode $(100 \mathrm{nl} / \mathrm{min})$. With an optical fiber $(200 \mu \mathrm{m}$ OD) touched on the brain surface, we illuminated a focal area $\sim 312 \mu \mathrm{m}$ OD to induce the local photorelease of 4-AP (Figure 2). As with the topical RuBi-4-AP application, there was no abnormal brain activity without blue light illumination after focal RuBi-4-AP injection (Figure 2B). However, repeated $1 \mathrm{~s}$ short duration blue light stimulations elicited interictal spikes and polyspikes. The average time from the start of the stimulation after such a short pulse to the occurrence of an interictal spike was $2.34 \pm 1.69$ s. Longer light illumination $(600 \mathrm{~s})$ induced seizure-like ictal discharges (Figures 2C,D). Additional photostimulations were given if there were no ictal discharges in $5 \mathrm{~min}$. We recorded 38 ictal discharges using focal illumination ( $n=5$ mice.) The average duration of seizures was $13.81 \pm 5.33 \mathrm{~s}$. The average time it took to record a seizure after a longer duration illumination onset was $638.32 \pm$
$138.82 \mathrm{~s}$ (See each mouse's ictal data in Supplementary Material Table S1).

In order to investigate whether focal illumination of the cortical surface would trigger events that start at the surface or deeper in lower cortical layers, we recorded RuBi-4-AP events using a multi-contact laminar depth electrode (Figure 3A). In the presence of RuBi-4-AP, a short 1 s duration pulse of photostimulation resulted in small events that were not full-formed interictal spikes but appeared to be elicited by a small population of neurons exhibiting a post-synaptic excitatory event (Figure 3B). Longer duration illumination (60s) resulted in the immediate onset of polyspike events occurring in all layers simultaneously that did not evolve into full blown seizures (Figures 3B-D). Layer-specific events sometimes occurred after the illumination was terminated and these events often exhibited field effects in all cortical layers (Figure 3E). However, most events involved all layers simultaneously (Figure 3F). Similar events were recorded in all animals ( $n=4$ mice). Of all the events ( $n=103$ events, 4 mice) recorded, $10.68 \%$ were layer-specific and $89.32 \%$ involved all layers simultaneously. Of the layer-specific events, all first started from layers $\mathrm{IV}$ to $\mathrm{V}$ and then propagated to the other neocortical layers.

\section{OPTICAL MAPPING OF RuBi-4-AP ICTAL DISCHARGE}

In order to determine if neurovascular coupling and propagation of the RuBi-4-AP events were similar to those caused by direct 4-AP injection, we performed optical spectroscopic mapping of the triggered events and compared them with maps derived from direct 4-AP injection. Optical recording of CBV at $530 \mathrm{~nm}$ during RuBi-4-AP ictal events demonstrated a monophasic increase in CBV (Figure 4A), with a mean latency of $0.82 \pm 0.53 \mathrm{~s}$. The mean duration of CBV increase was $9.58 \pm 1.74 \mathrm{~s}$. The maximum amplitude of focal CBV increase was $5.56 \pm 0.95 \%(n=5$ mice, 30 ictal discharges). At $780 \mathrm{~nm}$, the maps of light scattering demonstrated a monophasic increase as well but of smaller amplitude and spatial spread (Figure 4B). The mean duration of $780 \mathrm{~nm}$ signal increase was $8.38 \pm 2.57 \mathrm{~s}$. The maximum amplitude of focal $780 \mathrm{~nm}$ signal increase was $2.27 \pm 0.16 \%(n=5$ mice, 30 ictal discharges). Both CBV and light scattering imaging showed a clear optical response to RuBi-4-AP ictal events.

In order to compare RuBi-4-AP-induced seizures with seizures induced by injected standard 4-AP (Figure 5), we injected $0.5 \mu \mathrm{l}$ 4-AP ( $15 \mathrm{mM}$ ) into the neocortex as described previously (Zhao et al., 2011). The power of the resulting LFP seizure was $122.79 \pm$ $63.93 \mathrm{mV}^{2}$ ( $n=5$ mice, 30 ictal discharges) with RuBi-4-AP and $3414.75 \pm 836.83 \mathrm{mV}^{2}$ ( $n=5$ mice, 57 ictal discharges $)$ with 4-AP. Average seizure power induced by RuBi-4-AP was significantly lower than seizure power induced by injected 4-AP $(p<$ $0.01)$. The morphology of the CBV and light scattering responses to RuBi-4-AP were similar to injected 4-AP but smaller in size and amplitude, as would be expected based on the size of the electrographic events (Figures 4C,D). The maximum amplitudes of focal CBV increase and $780 \mathrm{~nm}$ maps resulting from injected 4 -AP were $10.12 \pm 1.63$ and $4.78 \pm 0.88 \%$, respectively $(n=5$ mice, 57 ictal discharges), which was significantly larger than the RuBi-4AP amplitudes ( $p<0.05$; Figures 5B,C). The average duration of injected 4-AP ictal discharges was $53.02 \pm 14.56 \mathrm{~s}$, which was significant longer than the average duration of RuBi-4-AP seizures 


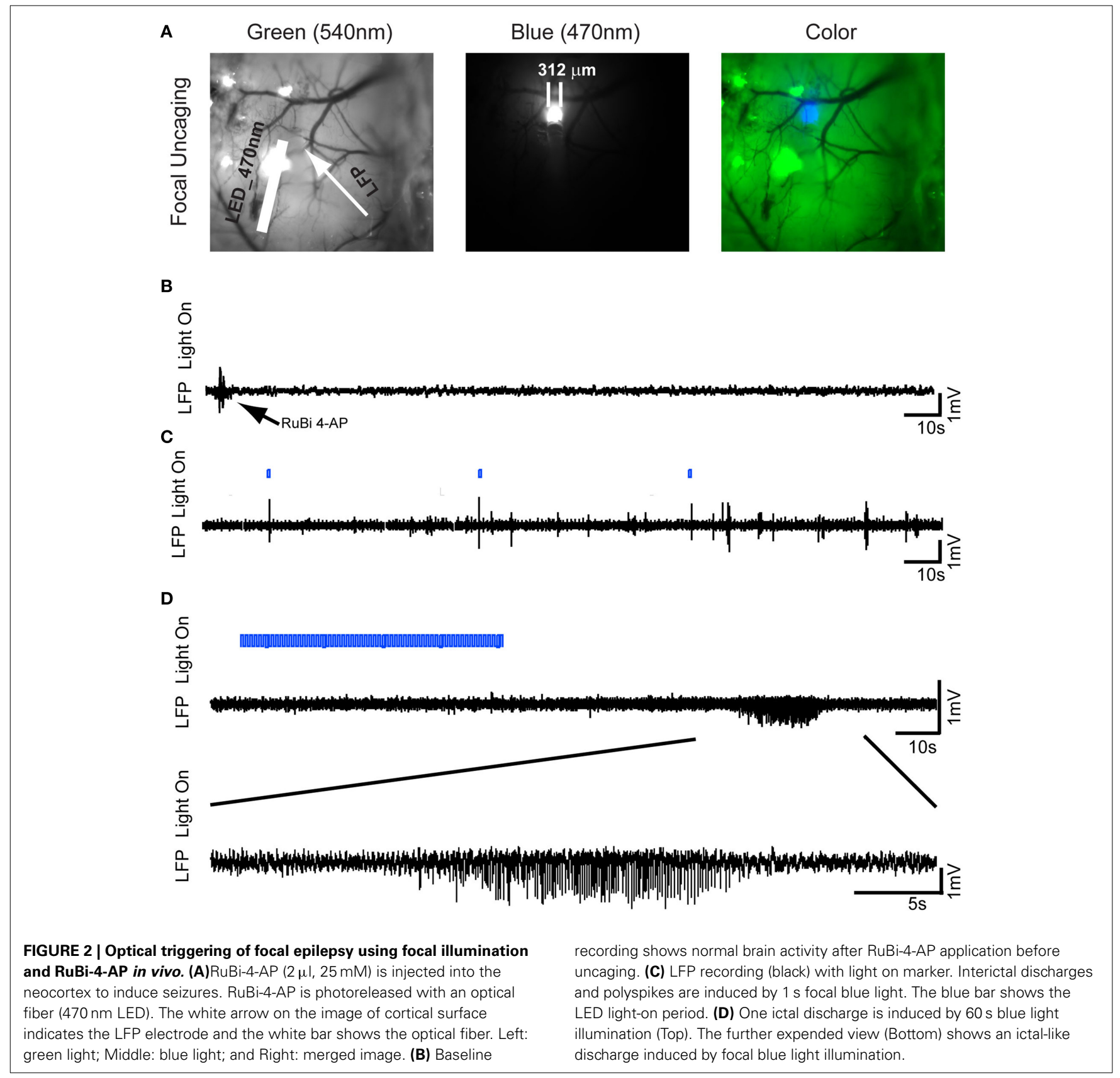

$(P<0.05$; Figure 5A). Thus, the hemodynamic and metabolic patterns of the RuBi-4-AP events indicate preserved neurovascular coupling, undamaged by any phototoxicity.

\section{DISCUSSION}

In this study, we provide, for the first time, evidence that photorelease of caged 4-AP can trigger epileptic events including interictal spikes, bursts of polyspikes, and ictal discharges of varying morphologies and durations. This model can be induced in vivo with both whole brain and focal illumination to provide high spatio-temporal control of the site of ictal onset. We describe two separate techniques for using of RuBi-4-AP and provide the foundation for other uses. With global application and wide-field illumination both interictal spikes and full-blown ictal events can be triggered. The former occur within seconds whereas the latter take a few minutes to develop. The location of the onset of these events is multifocal but the technique is non-invasive since the drug is applied topically. The variability in the resulting morphological of the electrographic events that can be triggered reflects the myriad of electrographic patterns manifested in clinical epilepsy (Prince, 2014). With focal injection, and focal illumination, the site of onset can be controlled more precisely. The amplitude and duration of these events vary based on the duration of illumination and can even occur in single layers, which provides not only the ability to investigate the development of epileptic events in a graded fashion but the occurrence 


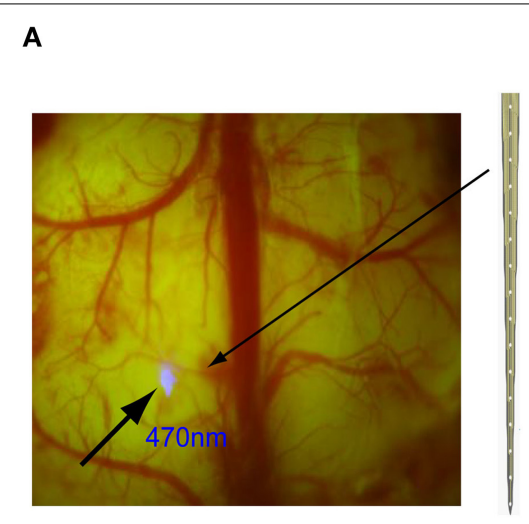

D

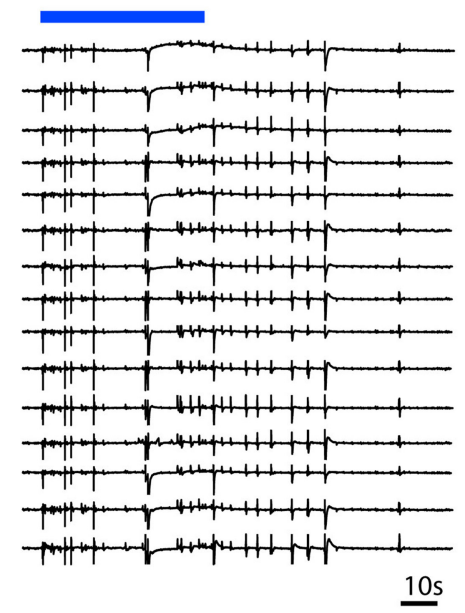

B

E

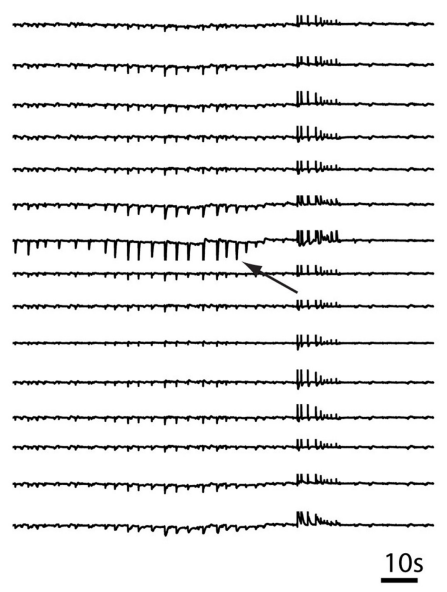

C
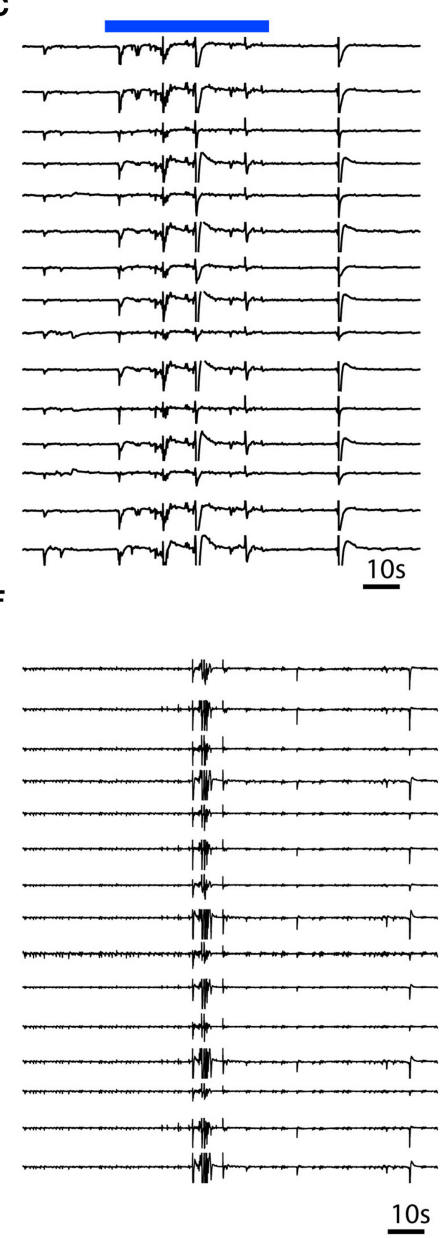

FIGURE 3 | Multi-contact laminar depth array recording of in vivo RuBi-4-AP epileptic events. The depth electrode is placed $0.2 \mathrm{~mm}$ away from the site of focal illumination. (A) Focal photostimulation $0.2 \mathrm{~mm}$ away from the laminar depth electrode placed tangentially into the cortex. (B) A $1 \mathrm{~s}$ pulsed photostimulation (blue line) results in a short duration event that occurred simultaneously in all layers. (C,D)
Longer duration illumination (60 s) results in immediate onset of polyspikes and short duration events involving all layers simultaneously. (E) Occasionally, layer specific events (black arrow) occur that quickly spread to all other layers. (F) However, the majority of events involve all layers simultaneously. The blue bars in $\mathbf{( B , C )}$ show the timing of blue photostimulation. of microseizures, which have been shown to occur in humans and whose synchronization may be critical to the development of human ictal events (Schevon et al., 2008; Stead et al., 2010). Recent data indicated that seizure initiation and spread is not highly hyper-synchronous (Truccolo et al., 2011). Using RuBi-4AP, the ability to alter the concentration of the drug as well as the size and amplitude of the illumination can lead to an almost infinite number of possibilities, which can be tuned to create precisely the desired type of epileptiform event. As such, the full range of combinatorial events is beyond the scope of this paper, as are the possibilities available with 2-photon illumination to trigger seizures in even smaller microdomains and specific layers as discussed below. As we have shown, this model is also useful to investigate neurovascular coupling as the amplitude and duration of events are shorter than would be produced with direct focal injection. Likewise, the resulting amplitude and duration of the associated vascular events are smaller, indicating proportional relationship in epileptiform neurovascular coupling, consistent with the existing literature (Bruehl et al., 1998; Ma et al., 2009; Geneslaw et al., 2011).

\section{OPTICALLY-TRIGGERED SEIZURES}

New neuroimaging and neuromodulatory techniques, such as optogenetics, which combine optical and genetic methods, and optochemistry, which involve the photorelease of active drugs, have emerged as popular tools to probe and control neuronal function with light (Zemelman et al., 2002; Lima and Miesenböck, 2005; Petreanu et al., 2007; Szobota et al., 2007; Zhang et al., 2007; Zayat et al., 2011; Chow and Boyden, 2013). Recently, optical suppression of epilepsy has been studied using both optogenetic techniques as well as caged compounds (Tonnesen et al., 2009; Yang et al., 2010). Optogenetics involves a gene manipulation, while caged methods involve a pharmacological manipulation of a chemical so that light can trigger the release 


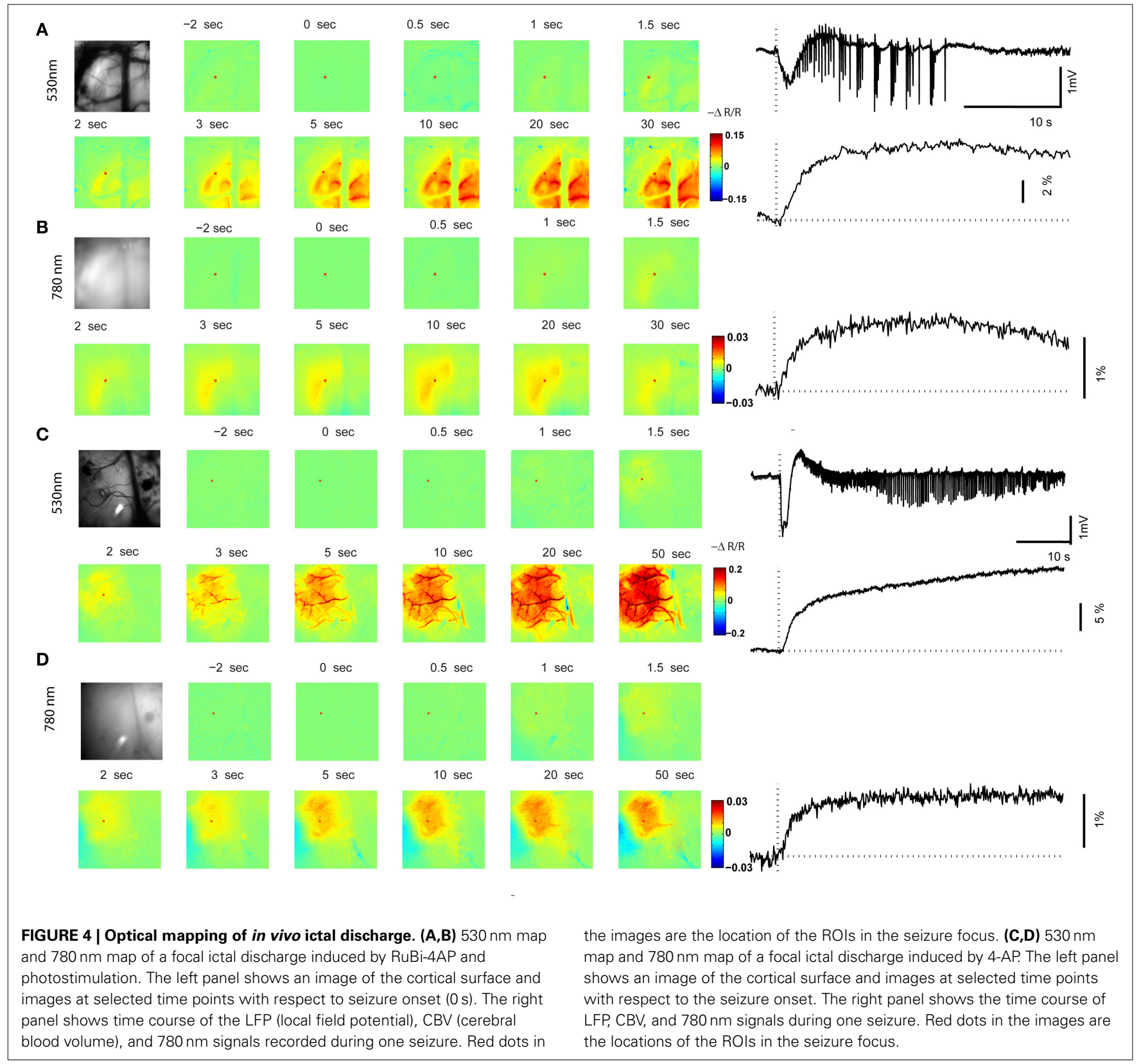

of the chemical. Unlike optogenetic methods, the photochemical approach does not require gene modification, which means the manipulation is reversible.

Acute rodent models of neocortical epilepsy include focal injection and topical application of pharmacologic agents. Focal GABAergic blockade is effective at creating interictal events in vivo but full blown seizures rarely occur (Schwartz and Bonhoeffer, 2001; Suh et al., 2005). 4-AP is a potent convulsant when applied to the neocortex. The microinjection of 4-AP can induce focal seizures, but the ability to trigger multiple sites of onset is limited and the seizures begin at a specific time after injection that cannot be controlled once the drug is injected. The possibility of optical triggering of interictal and ictal events offers several advantages in seizure control and modeling. Two methods currently exist for possible optical triggering of seizures, optogenetics and uncaging. While optogenetics holds certain advantages, such as the ability to target specific cell-types and both open and close channels thereby providing a reversible trigger (Fenno et al., 2011), the majority of research in the field has focused on seizure termination rather than seizure induction. Although unclear at this time, it may be that the specificity of optogenetics is too fine to reliably elicit seizures, which often requires a perturbation in a large network of cells. Recently, however, seizure-like afterdischarges were elicited optogenetically in in vivo hippocampus (Osawa et al., 2013). Uncaging, on the other hand, introduces a pharmacologic agent non-specifically which can influence the network at multiple levels and in multiple cell types. While less specific, 


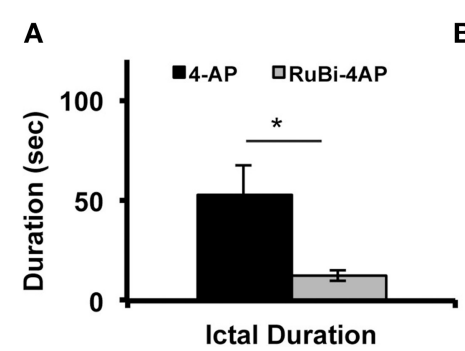

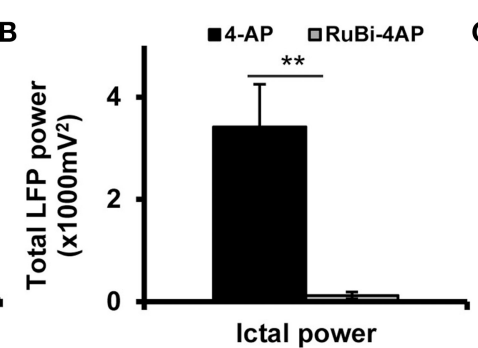

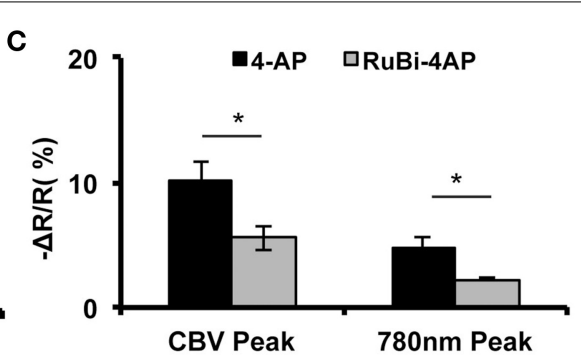

FIGURE 5 |CBV and light scattering response to ictal discharges elicited by RuBi-4-AP or 4-AP. (A) Average seizure duration induced by RuBi-4-AP is significantly shorter compared to seizure duration induced by 4-AP $(p<0.05)$. (B) Average total LFP power of seizures induced by RuBi-4-AP was significantly decreased compared with the total LFP power of seizures induced by 4-AP $(p<0.01) \quad$ (C) Average maximum response of CBV and $780 \mathrm{~nm}$ signals in the seizure focus was significantly decreased in the RuBi-4-AP compared with the 4-AP seizures (CBV: $P=0.04 ; 780 \mathrm{~nm}$ : $p=0.023) . * p<0.05 ; * * p<0.01$. this manipulation may be more efficacious at achieving the goal of epileptogenesis. It is also possible to induce focal seizures in deep brain regions, such as the hippocampus using optical fibers. Furthermore, unlike 4-methoxy-7-nitroindolinyl- (MNI) or 1-(2nitrophenyl)ethoxycarbonyl (NPEC) group caged compounds, which require UV light $(<360 \mathrm{~nm}), \mathrm{RuBi}$ - caged chemicals can be photo-triggered by visible blue light $(470 \mathrm{~nm})$, which has a higher tissue penetration and is less damaging to the living tissue. RuBi-4-AP also has a higher quantum efficiency of uncaging (Nikolenko et al., 2005). The lack of cell specificity is also an advantage at seizure termination, which has been successfully performed using RuBi-GABA (Yang et al., 2010, 2012).

\section{MICROSEIZURES AND LAMINA-SPECIFIC SEIZURES}

The ability to use RuBi-4-AP to elicit microseizures in multiple limited cortical domains can be aided with the development of light-delivery technology. One such technology, micro-LED arrays, permits multisite photostimulations (Grossman et al., 2010). Likewise, two-photon uncaging has been successfully used to activate individual neurons and dendritic spines (Fino et al., 2009). The application of multisite photostimulation in the RuBi4-AP model will provide the ability to model multiple microseizures. Using fine resolution microelectrode arrays in human epileptic tissue has identified a new class of electrographic seizures localized to sub-millimeter-scale tissue, at the level of the cortical column, labeled "microseizures" (Schevon et al., 2008; Stead et al., 2010). The existence of microseizures suggests that epileptiform activity can occur within extremely small neuronal networks. Microseizures play an important role in the initiation of epileptic seizures as small localized events appear to coalesce into full-blown seizures (Dudek, 2009). Most importantly, multiple small microseizures can occur in multiple regions of cortex either simultaneously or sequentially. Currently there is no model of microseizures in existence to investigate this novel phenomenon and RuBi-4-AP provides such a technique.

Using a depth laminar array electrode, we have also demonstrated that RuBi-4-AP can trigger laminar-specific epileptiform events. Data from in vitro slice preparations and in vivo human experiments have suggested that seizures may initiate in layer 5 and propagate in layers 2-3 (Gutnick et al., 1982; Silva et al., 1991; Sutor et al., 1994; Chesi and Stone, 1998; Telfian and Connors,
1998; Ulbert et al., 2004; Jin et al., 2006). Other studies, however, have raised the hypothesis that epileptiform events can be formed in several (probably all) neocortical layers or may arise from hyperconnected neurons in layer 2/3 neurons (Tsau et al., 1999; Beaumont et al., 2012). Preliminary analysis of our data indicates that even with topical release of RuBi-4-AP, all layer-specific events occurred in infragranular layers.

How can release of 4-AP in supragranular layers trigger seizures in layers 4 and 5 ? To estimate the release of caged chemicals, it is important to understand the transmission of visible light in brain tissue. Measurements and theoretical calculations of blue light penetration have been previously done in the brain tissue (Adamantidis et al., 2007; Aravanis et al., 2007; Huber et al., 2008; Yizhar et al., 2011). In our multi-contact laminar electrode array experiment, the tip of optical fiber was put on the top of exposed cortex. Due to the light absorption and scattering in brain tissue, the light-induced release of RuBi-4AP would be relatively well-localized at the surface of neocortex. Most neocortical neurons $(70-80 \%)$ are excitatory pyramidal neurons and the remaining $20-30 \%$ are interneurons, mostly inhibitory interneurons (Markram et al., 2004). Layer 1 consists primarily of the distal tufts of pyramidal cell apical dendrites, a few of GABAergic neurons, and many axon terminations. More specifically, the excitatory cells in layer 4 and 5 have their dendrites in the superficial layers. Layer 1 is a major target of -feedback- connections between associated cortical areas (Douglas and Martin, 2004). We hypothesize that topical release of 4-AP acts on the dendrites of the pyramidal cells in layers 4 and 5. Excitatory neurons in the neocortex use recurrent excitatory feedback as an effective network conductance to amplify external network inputs (Douglas et al., 1995). These large pyramidal neurons in layer 5 typically respond with bursting discharge patterns (Stoop et al., 2002). The epileptic discharges may then be triggered by increasing recurrent excitatory connections.

In conclusion, we provide a novel optically-triggered rodent epilepsy model using a caged chemical. Although the precise control of this RuBi-4-AP model is still under development, we have already shown that epileptiform events including interictal spikes, polyspikes, and ictal discharges were induced using a visual-range wavelength light illumination. Both multisite LFP recording and optical mapping showed clear seizure-like activity. 
Further development of this model in vivo and in vitro will be useful to address specific questions regarding epileptogenesis and neurovascular coupling.

\section{AUTHOR CONTRIBUTIONS}

Mingrui Zhao, Rafael Yuste and Theodore H. Schwartz generated the research idea, study design and concept. Mingrui Zhao, Rafael Yuste, and Theodore H. Schwartz wrote the manuscript. Mingrui Zhao, Laura M. McGarry, Hongtao Ma, and Samuel Harris performed experiments. Mingrui Zhao, Jason Berwick, Rafael Yuste, and Theodore H. Schwartz analyzed and interpreted data.

\section{ACKNOWLEDGMENTS}

All caged compounds were synthetized by the laboratory of Roberto Etchenique at the Universidad de Buenos Aires and we thank him for gifts of compounds and for his inspiration. This work was supported by the NINDS RO1 NS49482 (Theodore H. Schwartz), the Clinical and Translational Science Center (CTSC) Grant UL1 RR 024996 Pilot Grant (Mingrui Zhao), the Cornell University Ithaca-WCMC seed grant (Mingrui Zhao), and the Wellcome Trust [grant number: 093069] (Samuel Harris and Jason Berwick). The Rafael Yuste laboratory is supported by the NEI (DP1EY024503, R01EY011787), NIHM (R01MH101218, R21MH100646), NIDA (R21DA034195), DARPA contract W91NF-14-1-0269 and Keck Foundation. This material is based upon work supported by, or in part by, the U. S. Army Research Laboratory and the U. S. Army Research Office under contract number W911NF-12-10594 (MURI). We thank V Kanumuri, and D Peterka for help with uncaging experiments.

\section{SUPPLEMENTARY MATERIAL}

The Supplementary Material for this article can be found online at: http://www.frontiersin.org/journal/10.3389/fnins.2015. 00025/abstract

\section{REFERENCES}

Adamantidis, A. R., Zhang, F., Aravanis, A. M., Deisseroth, K., and De Lecea, L. (2007). Neural substrates of awakening probed with optogenetic control of hypocretin neurons. Nature 450, 420-424. doi: 10.1038/nature06310

Aravanis, A. M., Wang, L. P., Zhang, F., Meltzer, L. A., Mogri, M. Z., Schneider, M. B., et al. (2007). An optical neural interface: in vivo control of rodent motor cortex with integrated fiberoptic and optogenetic technology. J. Neural Eng. 4, S143-S156. doi: 10.1088/1741-2560/4/3/S02

Araya, R., Andino-Pavlovsky, V., Yuste, R., and Etchenique, R. (2013). Two-photon optical interrogation of individual dendritic spines with caged dopamine. ACS Chem. Neurosci. 4, 1163-1167. doi: 10.1021/cn4000692

Barkai, E., Friedman, A., Grossman, Y., and Gutnick, M. J. (1995). Laminar pattern of synaptic inhibition during convulsive activity induced by 4-aminopyridine in neocortical slices. J. Neurophysiol. 73, 1462-1467.

Beaumont, T. L., Yao, B., Shah, A., Kapatos, G., and Loeb, J. A. (2012). Layerspecific CREB target gene induction in human neocortical epilepsy. J. Neurosci. 32, 14389-14401a. doi: 10.1523/JNEUROSCI.3408-12.2012

Begley, C. E, Famulari, M., Annegers, J. F, Lairson, D. R, Reynolds, T. F, Coan, S., et al. (2000). The cost of epilepsy in the United States: an estimate from population-based clinical and survey data. Epilepsia 41, 342-351. doi: 10.1111/j.1528-1157.2000.tb00166.x

Benardo, L. S. (1997). Recruitment of GABAergic inhibition and synchronization of inhibitory interneurons in rat neocortex. J. Neurophysiol. 77, 3134-3144.

Bruckner, C., and Heinemann, U. (2000). Effects of standard anticonvulsant drugs on different patterns of epileptiform discharges induced by 4-aminopyridine in combined entorhinal cortex-hippocampal slices. Brain Res. 859, 15-20. doi: 10.1016/S0006-8993(99)02348-3

Bruehl, C., Hagemann, G., and Witte, O. W. (1998). Uncoupling of blood flow and metabolism in focal epilepsy. Epilepsia 39, 1235-1242. doi: 10.1111/j.15281157.1998.tb01320.x

Chesi, A. J. R., and Stone, T. W. (1998). Epileptiform activity in supragranular and infragranular blocks of mouse neocortex. Epilepsy Res. 31, 29-38. doi: 10.1016/S0920-1211(98)00013-8

Chow, B. Y., and Boyden, E. S. (2013). Optogenetics and translational medicine. Sci. Transl. Med. 5, 177ps175. doi: 10.1126/scitranslmed.3003101

De la Cruz, E., Zhao, M., Guo, L., Ma, H., Anderson, S., and Schwartz, T. (2011). Interneuron progenitors attenuate the power of acute focal ictal discharges. Neurotherapeutics 8, 763-773. doi: 10.1007/s13311-011-0058-9

Douglas, R. J., Koch, C., Mahowald, M., Martin, K. A., and Suarez, H. H. (1995). Recurrent excitation in neocortical circuits. Science 269, 981-985. doi: $10.1126 /$ science.7638624

Douglas, R. J., and Martin, K. A. (2004). Neuronal circuits of the neocortex. Annu. Rev. Neurosci. 27, 419-451. doi: 10.1146/annurev.neuro.27.070203.144152

Dudek, F. E. (2009). Microseizures in human neocortex: a role for ultra-small seizures? Epilepsy Curr. 9, 151-152. doi: 10.1111/j.1535-7511.2009.01325.x

Fenno, L., Yizhar, O., and Deisseroth, K. (2011). The development and application of optogenetics. Annu. Rev. Neurosci. 34, 389-412. doi: 10.1146/annurev-neuro061010-113817

Fino, E., Araya, R., Peterka, D. S., Salierno, M., Etchenique, R., and Yuste, R. (2009). RuBi-glutamate: two-photon and visible-light photoactivation of neurons and dendritic spines. Front. Neural Circuits 3:2. doi: 10.3389/neuro.04.002.2009

Frostig, R. D., Lieke, E. E., Ts'o, D. Y., and Grinvald, A. (1990). Cortical functional architecture and local coupling between neuronal activity and the microcirculation revealed by in vivo high-resolution optical imaging of intrinsic signals. Proc. Natl. Acad. Sci. U.S.A. 87, 6082-6086. doi: 10.1073/pnas.87.16.6082

Geneslaw, A. S., Zhao, M., Ma, H., and Schwartz, T. H. (2011). Tissue hypoxia correlates with intensity of interictal spikes. J. Cereb. Blood Flow Metab. 31, 1394-1402. doi: 10.1038/jcbfm.2011.16

Grinvald, A., Lieke, E., Frostig, R. D., Gilbert, C. D., and Wiesel, T. N. (1986). Functional architecture of cortex revealed by optical imaging of intrinsic signals. Nature 324, 361-364. doi: 10.1038/324361a0

Grossman, N., Poher, V., Grubb, S. M., Kennedy, T. G., Nikolic, K., Mcgovern, B., et al. (2010). Multi-site optical excitation using ChR2 and micro-LED array. J. Neural Eng. 7:016004. doi: 10.1088/1741-2560/7/1/016004

Gutnick, M. J., Connors, B. W., and Prince, D. A. (1982). Mechanisms of neocortical epileptogenesis in vitro. J. Neurophysiol. 48, 1321-1335.

Harris, S., Bruyns-Haylett, M., Kennerley, A., Boorman, L., Overton, P. G., Ma, H., et al. (2013). The effects of focal epileptic activity on regional sensory-evoked neurovascular coupling and postictal modulation of bilateral sensory processing. J. Cereb. Blood Flow Metab. 33, 1595-1604. doi: 10.1038/jcbfm.2013.115

Huber, D., Petreanu, L., Ghitani, N., Ranade, S., Hromadka, T., Mainen, Z., et al. (2008). Sparse optical microstimulation in barrel cortex drives learned behaviour in freely moving mice. Nature 451, 61-64. doi: 10.1038/nature06445

Jin, X., Prince, D. A., and Huguenard, J. R. (2006). Enhanced excitatory synaptic connectivity in layer v pyramidal neurons of chronically injured epileptogenic neocortex in rats. J. Neurosci. 26, 4891-4900. doi: 10.1523/JNEUROSCI.436105.2006

Lima, S. Q., and Miesenböck, G. (2005). Remote control of behavior through genetically targeted photostimulation of neurons. Cell 121, 141-152. doi: 10.1016/j.cell.2005.02.004

Löscher, W. (2011). Critical review of current animal models of seizures and epilepsy used in the discovery and development of new antiepileptic drugs. Seizure 20, 359-368. doi: 10.1016/j.seizure.2011.01.003

Ma, H., Zhao, M., and Schwartz, T. H. (2013). Dynamic neurovascular coupling and uncoupling during ictal onset, propagation, and termination revealed by simultaneous in vivo optical imaging of neural activity and local blood volume. Cereb. Cortex 23, 885-899. doi: 10.1093/cercor/bhs079

Ma, H., Zhao, M., Suh, M., and Schwartz, T. H. (2009). Hemodynamic surrogates for excitatory membrane potential change during interictal epileptiform events in rat neocortex. J. Neurophysiol. 101, 2550-2562. doi: 10.1152/jn.906 94.2008

Markram, H., Toledo-Rodriguez, M., Wang, Y., Gupta, A., Silberberg, G., and Wu, C. (2004). Interneurons of the neocortical inhibitory system. Nat. Rev. Neurosci. 5, 793-807. doi: 10.1038/nrn1519 
Mattia, D., Haw, G. G., and Avoli, M. (1993). Epileptiform activity induced by 4aminopyridine in guinea-pig and rat neocortices. Neurosci. Lett. 154, 157-160. doi: 10.1016/0304-3940(93)90195-Q

Mayer, G., and Heckel, A. (2006). Biologically active molecules with a "light switch." Angew. Chem. Int. Ed. Engl.45, 4900-4921. doi: 10.1002/anie.200600387

Navarro, V., Martinerie, J., Quyen, M. L. V., Clemenceau, S., Adam, C., Baulac, M., et al. (2002). Seizure anticipation in human neocortical partial epilepsy. Brain 125, 640-655. doi: 10.1093/brain/awf048

Nikolenko, V., Yuste, R., Zayat, L., Baraldo, L. M., and Etchenique, R. (2005). Twophoton uncaging of neurochemicals using inorganic metal complexes. Chem. Commun. (Camb.) 1752-1754. doi: 10.1039/b418572b

Osawa, S.-I., Iwasaki, M., Hosaka, R., Matsuzaka, Y., Tomita, H., Ishizuka, T., et al. (2013). Optogenetically induced seizure and the longitudinal hippocampal network dynamics. PLOS ONE 8:e60928. doi: 10.1371/journal.pone. 0060928

Petreanu, L., Huber, D., Sobczyk, A., and Svoboda, K. (2007). Channelrhodopsin2-assisted circuit mapping of long-range callosal projections. Nat. Neurosci. 10, 663-668. doi: 10.1038/nn1891

Prince, D. (2014). "How do we make models that are useful in understanding partial epilepsies?", in Issues in Clinical Epileptology: A View from the Bench, eds. H. E. Scharfman and P. S. Buckmaster (Netherlands: Springer), 233-241. doi: 10.1007/978-94-017-8914-1_18

Rial Verde, E. M., Zayat, L., Etchenique, R., and Yuste, R. (2008). Photorelease of GABA with Visible Light Using an Inorganic Caging Group. Front. Neural Circuits 2:2. doi: 10.3389/neuro.04.002.2008

Rogawski, M. A., and Barker, J. A. (1983). Effects of 4-aminopyridine on calcium action potentials and calcium current under voltage clamp in spinal neurons. Brain Res. 280, 180-185. doi: 10.1016/0006-8993(83)91190-3

Salierno, M., Marceca, E., Peterka, D. S., Yuste, R., and Etchenique, R. (2010). A fast ruthenium polypyridine cage complex photoreleases glutamate with visible or IR light in one and two photon regimes. J. Inorg. Biochem. 104, 418-422. doi: 10.1016/j.jinorgbio.2009.12.004

Salzberg, B. M., Obaid, A. L., and Gainer, H. (1985). Large and rapid changes in light scattering accompany secretion by nerve terminals in the mammalian neurohypophysis. J. Gen. Physiol. 86, 395-411. doi: 10.1085/jgp. 86.3.395

Schevon, C. A., Ng, S. K., Cappell, J., Goodman, R. R., Mckhann, G. J., Waziri, A., et al. (2008). Microphysiology of epileptiform activity in human neocortex. J. Clin. Neurophysiol. 25, 321-330 doi: 10.1097/WNP.0b013e31818e8010

Schwartz, T. H., and Bonhoeffer, T. (2001). In vivo optical mapping of epileptic foci and surround inhibition in ferret cerebral cortex. Nat. Med. 7, 1063-1067. doi: 10.1038/nm0901-1063

Silva, L. R., Amitai, Y., and Connors, B. (1991). Intrinsic oscillation of neocortex generated by layer 5 pyramidal neurons. Science 251, 432-435. doi: $10.1126 /$ science. 1824881

Stansfeld, C. E., Marsh, S. J., Halliwell, J. V., and Brown, D. (1986). 4Aminopyridine and dendrotoxin induce repetitive firing in rat visceral sensory neurons by slowly inactivating outward current. Neurosci. Lett. 64, 299-304. doi: 10.1016/0304-3940(86)90345-9

Stead, M., Bower, M., Brinkmann, B. H., Lee, K., Marsh, W. R., Meyer, F. B., et al. (2010). Microseizures and the spatiotemporal scales of human partial epilepsy. Brain 133, 2789-2797. doi: 10.1093/brain/awq190

Stoop, R., Blank, D., Kern, A., van der Vyver, J.-J., Christen, M., and Wagner, C. (2002). Collective bursting in layer IV: synchronization by small thalamic inputs and recurrent connections. Cogn. Brain Res. 13, 293-304. doi: 10.1016/S09266410(01)00123-9

Suh, M., Bahar, S., Mehta, A. D., and Schwartz, T. H. (2005). Temporal dependence in uncoupling of blood volume and oxygenation during interictal epileptiform events in rat neocortex. J. Neurosci. 25, 68-77. doi: 10.1523/JNEUROSCI.282304.2005

Sutor, B., Hablitz, J. J., Rucker, F., and Bruggencate, G. (1994). Spread of epileptiform activity in the immature rat neocortex studied with voltage-sensitive dyes and laser scanning microscopy. J. Neurophys. 4, 1756-1768.

Szobota, S., Gorostiza, P., Del Bene, F., Wyart, C., Fortin, D. L., Kolstad, K. D., et al. (2007). Remote control of neuronal activity with a light-gated glutamate receptor. Neuron 54, 535-545. doi: 10.1016/j.neuron.2007.05.010

Telfian, A. E., and Connors, B. W. (1998). Layer-specific pathways for the horizontal propagation of epileptiorm discharges in neocortex. Epilepsia 39, 700-708. doi: 10.1111/j.1528-1157.1998.tb01154.x
Thurman, D. J., Beghi, E., Begley, C. E., Berg, A. T., Buchhalter, J. R., Ding, D., et al. (2011). Standards for epidemiologic studies and surveillance of epilepsy. Epilepsia 52, 2-26. doi: 10.1111/j.1528-1167.2011. 03121.x

Tonnesen, J., Sorensen, A. T., Deisseroth, K., Lundberg, C., and Kokaia, M. (2009). Optogenetic control of epileptiform activity. Proc. Natl. Acad. Sci. U.S.A. 106, 12162-12167. doi: 10.1073/pnas.0901915106

Truccolo, W., Donoghue, J. A., Hochberg, L. R., Eskandar, E. N., Madsen, J. R., Anderson, W. S., et al. (2011). Single-neuron dynamics in human focal epilepsy. Nat. Neurosci. 14, 635-641. doi: 10.1038/nn.2782

Tsau, Y., Guan, L., and Wu, J.-Y. (1999). Epileptiform activity can be initiated in various neocortical layers: an optical imaging study. J. Neurophysiol. 82, 1965-1973.

Ulbert, I., Heit, G., Madsen, J., Karmos, G., and Halgren, E. (2004). Laminar analysis of human neocortical interictal spike generation and propagation: current source density and multiunit analysis in vivo. Epilepsia 45, 48-56. doi: 10.1111/j.0013-9580.2004.04011.x

Wilner, A. N., Sharma, B. K., Thompson, A., Soucy, A., and Krueger, A. (2014). Diagnoses, procedures, drug utilization, comorbidities, and cost of health care for people with epilepsy in 2012. Epilepsy Behav. 41, 83-90. doi: 10.1016/j.yebeh.2014.08.131

Yang, X. F., Schmidt, B. F., Rode, D. L., and Rothman, S. M. (2010). Optical suppression of experimental seizures in rat brain slices. Epilepsia 51, 127-135. doi: 10.1111/j.1528-1167.2009.02252.x

Yang, X., Rode, D. L., Peterka, D. S., Yuste, R., and Rothman, S. M. (2012). Optical control of focal epilepsy in vivo with caged $\gamma$-aminobutyric acid. Ann. Neurol. 71, 68-75. doi: 10.1002/ana.22596

Yizhar, O., Fenno, L. E., Davidson, T. J., Mogri, M., and Deisseroth, K. (2011). Optogenetics in neural systems. Neuron 71, 9-34. doi: 10.1016/j.neuron.2011.06.004

Zayat, L., Baraldo, L. M., and Etchenique, R. (2011). "Uncaging with visible light: inorganic caged compounds," in Imaging: A Laboratory Manual, ed. R. Yuste. (Cold Spring Harbor, NY: Cold Spring Harbor Laboratory Press), 897-902.

Zemelman, B. V., Lee, G. A., Ng, M., and Miesenböck, G. (2002). Selective photostimulation of genetically ChARGed neurons. Neuron 33, 15-22. doi: 10.1016/S0896-6273(01)00574-8

Zhang, F., Wang, L.-P., Brauner, M., Liewald, J. F., Kay, K., Watzke, N., et al. (2007) Multimodal fast optical interrogation of neural circuitry. Nature 446, 633-639. doi: 10.1038/nature05744

Zhao, M., Ma, H., Suh, M., and Schwartz, T. H. (2009). Spatiotemporal dynamics of perfusion and oximetry during ictal discharges in the rat neocortex. J. Neurosci. 29, 2814-2823. doi: 10.1523/JNEUROSCI.4667-08.2009

Zhao, M., Nguyen, J., Ma, H., Nishimura, N., Schaffer, C. B., and Schwartz, T. H. (2011). Preictal and Ictal neurovascular and metabolic coupling surrounding a seizure focus. J. Neurosci. 31, 13292-13300. doi: 10.1523/JNEUROSCI.259711.2011

Zhao, M., Suh, M., Ma, H., Perry, C., Geneslaw, A., and Schwartz, T. H. (2007). Focal increases in perfusion and decreases in hemoglobin oxygenation precede seizure onset in spontaneous human epilepsy. Epilepsia 48, 2059-2067. doi: 10.1111/j.1528-1167.2007.01229.x

Conflict of Interest Statement: The authors declare that the research was conducted in the absence of any commercial or financial relationships that could be construed as a potential conflict of interest.

Received: 08 October 2014; accepted: 14 January 2015; published online: 04 February 2015.

Citation: Zhao M, McGarry LM, Ma H, Harris S, Berwick J, Yuste R and Schwartz TH (2015) Optical triggered seizures using a caged 4-Aminopyridine. Front. Neurosci. 9:25. doi: 10.3389/fnins.2015.00025

This article was submitted to Brain Imaging Methods, a section of the journal Frontiers in Neuroscience.

Copyright (c) 2015 Zhao, McGarry, Ma, Harris, Berwick, Yuste and Schwartz. This is an open-access article distributed under the terms of the Creative Commons Attribution License (CC BY). The use, distribution or reproduction in other forums is permitted, provided the original author(s) or licensor are credited and that the original publication in this journal is cited, in accordance with accepted academic practice. No use, distribution or reproduction is permitted which does not comply with these terms. 